\title{
Lungenerkrankungen unter den Top 10 der Todesursachen
}

Die Weltgesundheitsorganisation (WHO), die Weltbank und die School of Public Health der Harvard University haben 1991 ein großes Projekt ins Leben gerufen, das sich weltweit zur Aufgabe gemacht hat, Krankheiten, Todesfälle und den Grad der Behinderung durch Krankheiten sowie deren Risikofaktoren zu analysieren. Die aktuellsten Daten der Global-Burden-of-Disease-Studie wurden in der August-Ausgabe 2013 des New England Journal of Medicine und in The Lancet Ende 2012 veröffentlicht. Die Deutsche Gesellschaft für Pneumologie und Beatmungsmedizin e.V. (DGP) nimmt dies zum Anlass, auf einige wichtige Fakten hinzuweisen, die in der Öffentlichkeit häufig nicht in der angemessenen Weise wahrgenommen werden.

Der vor fast 20 Jahren prognostizierte Anstieg der weltweiten Todesursachenstatistik mit der chronisch obstruktiven Lungenerkrankung (COPD) auf nunmehr Platz 3 ist tatsächlich eingetreten. Lungenkrebs ist in dieser Zeitspanne von Platz 8 auf Platz 5 geklettert. Auf Platz 4 liegt die Lungenentzündung und auf Platz 10 die Tuberkulose. Somit stellen Erkrankungen der Lunge und des Atmungssystems weltweit einen großen Anteil der Todesursachen. Unter den 10 häufigsten Todesursachen in den USA finden sich ebenfalls 3 Lungenerkrankungen: An 3. Stelle steht nach ischämischer Herzerkrankung und Schlaganfall der Lungenkrebs, an 5. Stelle COPD und an 7. Stelle die Lungenentzündung. Betrachtet man den Grad der Einschränkung der Le- bensqualität durch eine Krankheit, die durch einen rechnerischen Index ausgedrückt wird (sog. Disability adjusted Life Years, so landet COPD sogar an 2. Stelle. Beunruhigend ist, dass sich die Qualität der Versorgung von COPD-Patienten zwischen 1991 und 2010 zumindest in den USA verschlechtert hat. Doch nicht nur in den sog. High Income Countries nehmen Erkrankungen der Lunge einen sehr wichtigen Platz ein, sondern weltweit. Der Grad der eingeschränkten Lebensqualität durch Lungenentzündung, COPD, Tuberkulose und Lungenkrebs ist im internationalen Vergleich sehr hoch. Hier liegt COPD noch deutlich vor Diabetes mellitus oder Eiweißmangel als Folge einer Fehlernährung.

Auch die bekannten Risikofaktoren für Krankheiten wurden analysiert. Tabakrauch, selbst als sog. Passivrauchen ein Risikofaktor für COPD und Lungenkrebs, findet sich an Platz 2 der wesentlichen Risikofaktoren (nach hohem Blutdruck). Die Zahl der Raucher nimmt in den Entwicklungsländern weiter zu, insbesondere in Ostasien. Die DGP nimmt die Ergebnisse dieser außerordentlich aufwändigen und einflussreichen Untersuchung, an der 486 Wissenschaftler in 301 Institutionen aus 50 Ländern über mehrere Jahre gearbeitet haben, zum Anlass, verstärkt auf die Bedeutung von Lungenerkrankungen und die Defizite in der Versorgung von Patienten mit Lungenerkrankungen hinzuweisen. Maßnahmen zur Bekämpfung des Tabakkonsums und der weiteren Verbesserung der Luftqualität dürfen trotz erster positiver Entwicklungen in Deutschland nicht nachlassen. Zur Entwicklung neuerer und besserer Therapien für COPD, Asthma, Lungenkrebs oder Lungenentzündungen sind weiterhin intensive Anstrengungen erforderlich. Hierzu muss die Pneumologie auch als Fach stärker in der deutschen Versorgungsstruktur verankert werden. An vielen Kliniken fehlen spezialisierte pneumologische Abteilungen. An Universitäten ist die Lungenheilkunde nur in lediglich 9 der 36 Medizinischen Fakultäten mit eigenständigen Professuren vertreten. Deutschland ist in dieser Hinsicht eines der Schlusslichter in Europa.

Demgegenüber steht die erfolgreiche Etablierung der 6 Deutschen Zentren der Gesundheitsforschung, zu denen das Deutsche Zentrum für Lungenforschung mit 5 Partnerstandorten gehört. Eine verbesserte Vorsorge und Diagnose sowie individualisierte Therapien und eine optimale Versorgung der Patienten stehen bei diesem Forschungsverbund im Vordergrund. Zentrales Ziel ist die Erforschung der wichtigen Lungenerkrankungen: Asthma und Allergie, COPD, zystische Fibrose, Lungenentzündung, akutes Lungenversagen in der Intensivmedizin, Lungenfibrose, Lungenhochdruck, Lungenkrebs. Diese Erkrankungen können zu einem chronischen Lungenversagen führen und eine Transplantation erforderlich machen.

Die DGP wird zur Situation der Pneumologie in Deutschland in Kürze eine Neuauflage des Weißbuchs Lunge publizieren. Auf dem Kongress der European Respiratory Society im September ist bereits das europäische Weißbuch zu Lungenerkrankungen erschienen.

Mitteilung der DGP, Berlin 